\title{
The Problem of infertility with Hyperprolactenemia
}

\author{
Samjahana Dhakal \\ Paropakar Maternity and Womens Hospital, \\ Thapathali, Kathmandu, Nepal
}

\begin{abstract}
Hyperprolactenemia is the most common endocrine disorder of the hypothalamic-pituitary axis. It is a common condition and the causes for it varies from physiological causes, use of medication, hypothyroidism as well as pituitary tumors. The main consequences are amenorrhea and infertility in women. There are some specific indications for treatment. The underlying cause, sex,age and reproductive status must be considered. Serum prolactin level measurement and radioimaging are the diagnostic method to diagnose hyperprolactenaemia. The aim of treatment are to normalize prolactin level,restore ovulation and reduce the effects of chronic Hyperprolactenemia. Dopamine agonists are the treatment of choice. Transphenoidal surgery is usually reserved for patients who are intolerant or resistant to dopamine agonists. Cabergoline is effective and better tolerated than bromocriptine, but it has been proven that bromocriptine is a safer drug, therefore it remains the treatment of choice in Hyperprolactenemia women who wants to conceive.
\end{abstract}

Keywords: Hyperprolactenemia, pituitary tumor, transphenoidal surgery

\section{Introduction}

hyperprolactenaemia is defined as an elevation of prolactin level above $25 \mathrm{ng} / \mathrm{ml}$ in women of childbearing age and above $20 \mathrm{ng} / \mathrm{ml}$ in men and postmenopausal women. ${ }^{1}$

It is a common condition with varied etiology. It is common in women,but also seen in men \& even in adolescence $\&$ child hood. ${ }^{2}$

It is the most common endocrine disorder of the hypothalamic-pituitary axis. The clinical syndrome resulting from hyperprolactenaemia has been recognized in women since ancient times, the biochemical condition is a relatively new disorder as human prolactin was only purified and verified to be distinct from human growth hormone in $1971 .{ }^{3}$

Prolactin is mainly a lactogenic hormone but it has other action also. Some recent data suggests that prolactin may have an added immuno modulatory role. ${ }^{4,5}$

Hyperprolactenaemia is mainly due to enlargement of the pituitary gland or due to a pituitary tumour or can occur without any obvious reason. ${ }^{6}$
Hypothalamus has inhibitory action in prolactin secretion. The inhibiting agent of hypothalamus is identified as dopamine. Anything that suppresses dopamine secretion or its transfer can lead to hyperprolactenaemia. ${ }^{7,8}$ Any drugs that stimulates or increase the action of dopamine can reduce the level of prolactin to normal.

\section{Epidemiology}

The prevalence ranges from $0.4 \%$ in an unselected healthy adult population in japan to $5 \%$ among clients at a family planning clinic. 9

\section{Effects of hyperprolactenaemia}

On hypothalamus, it decreases GnRH. On pituitary it decreases FSH and LH. On ovaries it decreases oestrogen and progesterone. Therefore follicles become abnormal. Luteal phase becomes abnormal Menstrual abnormality \& amenorrhoea occurs ${ }^{10}$

\section{Etiology}

Physiological increase in prolactin occurs during pregnancy, postpartum breastfeeding and stress. Prolactinomas account for 25 to $30 \%$ functioning

Correspondence

Dr Samjhana Dhakal, MD

Obstetrician \& Gynaecologist

Maternity Hospital, Thapathali Kathmandu,Nepal

Mobile: 9851066093

E-mail:dhakal_samjhana@hotmail.com 
pituitary tumors and are the most frequent causes of chronic hyperprolactenaemia. Other causes are non hypothalamic pituitary disease. Polycystic ovarian syndrome, Primary hypothyroidism, liver disease and chronic renal disease. Forty percent of patients of hypothyroidism have mild elevation of prolactin level. Some drugs like metoclopramide, phenothiazines, oestrogen and cimetidine can cause hyperprolactenaemia

\section{Prolactinomas}

Prolactin-secreting pituitary adenomas are generally benign and tend not to progress. When asymptomatic they may be followed by clinical and laboratory methods. When symptomatic they usually respond promptly to dopamine agonists (lowering of prolactin and shrinkage of tumour). In approximately $30 \%$ of individuals, with or without a visible microadenoma, the condition resolves spontaneously. ${ }^{11}$

Microadenoma are more common than macroadenoma. $15 \%$ of prolactinoma patients are diagnosed in postpartum period. The incidence of pituitary tumour is $20 \%$ with $50 \mathrm{ng} / \mathrm{ml}$ prolactin $\& 50$ to 100 $\%$ with $>100 \mathrm{ng} / \mathrm{ml}$. ${ }^{12}$ Therefore it is suggested that all patients who have prolactin level of $50 \mathrm{ng} / \mathrm{ml}$ should have radiological evaluation. ${ }^{13}$ In primary amenorrhoea 5-7 \% have prolactinoma where as in secondary amenorrhoea $23 \%$ have prolactinoma. ${ }^{14}$. Prolactinomas cause hyperprolactenaemia by increased secretion of prolactin. But other tumour in the base of the brain e.g craniopharyngioma can also cause hyperprolactenaemia by pressing on the stalk of pituitary \& thus preventing dopamine to act on hypothalamus to pituitary. ${ }^{6}$ In the presence of prolactinomas, prolactin remain the same during sleep also.

Some develop galactorrhoea \& amenorrhoea while on OC Pills These are undiagnosed prolactinomas. About $30 \%$ of women with post pill amenorrhoea have adenomas. Establishment of galactorrhoea while on OC Pills mandate prolactin determination. ${ }^{15}$

\section{Clinical features}

- Occur often without symptoms.

- Galactorrhoea may or may not be present

- Amnerrhoea and other menstrual dysfunction may or may not be present

- Decreased libido,Hirsutism,Acne may or may not be present.

- Infertility is often present.

- Anovulation may be present.

- Inadequate luteal phase \& the early abortion is one of the presenting feature.

- Primary hypothyroidism is often associated with hyperprolactenaemia.
- Pituitary adenoma (prolactinomas micro \& macro form $30 \%$ of the cause of hyperprolactenaemia). But only huge adenoma can cause symptomps like headache and visual field defects. ${ }^{16}$

- Progressive trabecular osteopenia in women with hyperprolactenemic amenorrhea may or may not be present. ${ }^{17}$

\section{Diagnostic Evaluation}

\section{Prolactin}

Prolactin level is obtained in a patient with specific clinical presentation like amenorrhea,galactorrhea and infertility. An initial level above the normal range should be repeated by drawing blood in the morning with the patient in a fasting state.

Normal level of prolactin is $5-25 \mathrm{ng} / \mathrm{ml}$.

Level more than $25 \mathrm{ng} / \mathrm{ml}$ mandates investigations.

Other factor which increases the prolactin level are sleep, this increases prolactin 2-3 times.

Food, Stress, coitus also increases prolactin

Several drugs that increases prolactin are: antihistam inic,tranquilizer, antiemetics, antihypertensive, neuroleptics(chlorpromazine, haloperidole) ${ }^{18}$

Estimation of TSH \&T4 -shows hypothyroidism

Radiological imaging of Sellaturcica, especially when level of prolaction $>50 \mathrm{ng} / \mathrm{ml}$

CT Scan

MRI-best visualization of the sellar area,especially if there is microadenoma $<10 \mathrm{~mm}$ diameter

$\mathrm{X}$-rays -shows osteoporosis

\section{Metoclopromide test}

This test possesses the diagnostic value and may be useful especially in cases ith marked basal level of prolactin.

In D20-22 of cycle, $10 \mathrm{mg}$ of inj metoclopromide is given and blood is checked. In functional hyperprolactenemia more than 25 fold increase of over basal values, unlike as in organic hyperprolactenemia. ${ }^{19}$

TRH test

D3-5of cycle with TRH 200/ugm injection. In a normal subject PRL level rises at least twice as high as basal value, but in hyperprolactenaemia, galactorrhoea or thyroid dysfunction PRL level does not rise. ${ }^{20}$

Visual field examination is necessary in the macroadenoma which has supracellar extension.

\section{Management}

All hyperprolactenemia does not require therapy. Withdraw Causative agent.

Treat hypothyroidism if present. Recent therapeutic options include Observation, medical therapy,surgery and radiotherapy. Men with microadenoma should be treated when they have hypogonadism, decreased potency, libido or infertility. 
Indications for therapy in hyperprolactenemia

- Infertility

- Amenorrhoea with osteoporosis

- Acne

- Hirsuitism

- $\quad$ Decreased libido

- Galactorrhoea

\section{Observation}

Idiopathic hyperprolactenemic patient who do not wish to conceive and who don't show signs of hyperprolactenemia may be observed with serial prolactin level.

The natural history of untreated prolactinomas remains unclear but the available evidence suggests that the risk of microprolactinoma to a macroprolactinoma is low that is under $10 \% .^{21}$ The female patients with small tumors and if there is no indications for treatment can be carefully monitored with serial prolactin level.

\section{Medical treatment}

Dopamine agonists are the treatment of choice.

Bromocriptine It is the first dopamine agonist introduced in clinical practice. It is a semisynthetic ergot derivative that binds to and stimulate dopamine $\left(\mathrm{D}_{2}\right)$ receptors on normal andadenomatous lactotroph cells. $^{22}$

It is effective in normalizing serum prolatin levels and restoring gonadal function in $80 \%$ to $90 \%$ of patients. Dr Moltich stated that data from 112 patients in 21 different studies showed that use of bromocriptine reduced the size of tumors by $50 \%$ or more in $40 \%$ of patients and by $25 \%$ to $50 \%$ in $25 \%$ of patients. In microadenoma the drug rapidly lowers serum prolacin level to normal in up to $70 \%$ of patients,decrease tumor size and restores gonadal function. In patients with macroadenomas,prolactin levels are also normalized in $70 \%$ of patients and tumor mass shrinkage $(>50 \%)$ is achieved in up to $40 \%$ of patients. ${ }^{23}$

Bromocriptine is the treatment of choice \& with good results. Ovulation rate is $90 \%$ \& pregnancy rate is $83 \%$.

\section{Dose Schedule}

$1 / 2$ tablets (1. 25mg)at bed time for 2-4 wks then increase the dose to $2.5 \mathrm{mg}$. Response is assessed by plasma oestradiol, progesterone, serum prolactin level. In the absence of response dose is doubled to $5 \mathrm{mg}, 2$. $5 \mathrm{mg}$ thrice daily. The most common adverse effects are nausea and vomiting. Dizziness can result in 25\% of patients. Other side effects are headache, auditary hallucinations, delusions and mood swings. ${ }^{24}$
This drug is can be used through intravaginal route. Effect of drug lasts upto $24 \mathrm{hrs}$ after a single dose and has less gastrointestinal side effects than with the oral route. ${ }^{20}$

In the absence of labarotary facilities, the dose can be increased empirically until response is seen by return of menstruation or pregnancy.

Prolactin level rapidly falls down with bromocriptine. Galactorrhoea also subsides. But ovarian activities does not commence until serum prolactin level goes below $10 \mathrm{ng} / \mathrm{ml}$. Usually the initial ovulation is not normal because of of follicular development without ovulation or ovulation with deficient corpus luteum function. Most of the patient conceive within six ovulatory cycles. Once ovulation occurs during treatment regular ovulation occurs as long as drug is administered. Drug taking should be stopped after positive pregnancy test. But prolactin rises up more than normal in pregnancy as it does not affect the foetus.

In $5 \%$ of patients tab clomiphane should be given for ovulation. In another $5 \%$ of hyperprolactinaemia larger doses of bromocriptine, even up to $30 \mathrm{mg} /$ day is required. ${ }^{23}$

If Ovarian function does not esablish with this dose then clomiphane citrate 100 to $150 \mathrm{mg}$ /day for 5 days is given. Bromocriptine should be continued during pregnancy with macro \& microadenoma, because these tumour may enlarge during pregnancy. ${ }^{25}$

\section{Cabergoline}

It is an ergoline derivative with high affinity and selectivity for the $\mathrm{D}_{2}$ receptor. It has long half life 65 hrs so dose is once or twice weekly administration. Serum prolactin level comes to normal in 85 to $86 \%$ and restoration of normal gonadal function in 90 to $91 \%$ in hyperprolactinemic patients after treatment. ${ }^{26}$

In a multicenter collaborative study Cabergoline and bromocriptine have been compared. ${ }^{27}$ Stable normoprolactinemia was achieved in $83 \%$ women in cabergoline group ,59\% women in the bromocriptine group. Cabergoline was better tolerated compared with bromocriptine.

\section{Oestrogen}

Oestrogen therapy in the form of hormone replacement therapy or an oral contraceptive may be offered as an alternative to dopamine agonist therapy to women with idiopathic hyperprolactenemia or microprolactinoma who do not want to become pregnant and in whom oestrogen deficiency is major concern. ,particularly in resistant cases of dopamine agonist or who are intolerant to it. ${ }^{20}$ Patient receiving oestrogen rather than a dopamine agonist should be monitored for symptoms or signs of tumor and discontinued if there is radiological evidence of tumor enlargement. 
Surgery Controversial and depends on size of tumour.

Transsphenoidal surgery is the preferred therapy for prolactinomas before the availability of of dopamine agonists Surgery has gradually been used less frequently as the primary therapy and is now usually reserved for unresponsive or intolerant of optimal medical therapy. ${ }^{28}$

The Success rate of surgery depends on the skill and experience of the surgeon. In a recent series of of 219 women with prolactinomas cure rate of up to $91 \%$ were achieved in women with intrasellar microadenomas and $59 \%$ in cavernous sinus extension. Morbidity and mortality rates of surgery vary considerably from center to center and have improved in recent years due to better imaging. ${ }^{28}$ The most common complication is hypopituitarism.

A low postoperative prolactin value measured 1 to 2 days after surgery appears to be the best predictor. Immeasurable serum prolactin $(<2 \mathrm{ng} / \mathrm{ml}$ or, $40 \mathrm{mu} / \mathrm{L})$ predicts a cure with more than $90 \%$ probability. ${ }^{29}$

\section{Radiotherapy}

Fractionated radiotherapy is now rarely used as a primary therapy in prolactinomas, not only because of complications but also because of poor results. ${ }^{30}$

\section{Monitoring and follow-up}

Clinical and biochemical improvements after dopamine agonist therapy are readily apparent in most patients. In addition, tumour shrinkage can be expected in about $80 \%$ of macroadenomas. ${ }^{31}$ However, a major drawback of medical therapy is the potential need for lifelong treatment. Discontinuation of bromocriptine therapy can lead to recurrence of hyperprolactenemia in most patients and to tumour regrowth if treatment duration has been less than 2 years. Passos and associates has reported normal prolactin levels and absence of adenoma re-expansion after withdrawal of dopamine agonist therapy in $6.6 \%$ to $37.5 \%$ of patients. ${ }^{32}$ Recurrence usually occurs within months after withdrawal of drug. These authors have also reported reduced and normal prolactin levels after pregnancy in women who had prolactinomas treated with dopamine agonists. Menopause has also been suggested as a condition that maintains normoprolactinemia after dopamine agonist therapy is stopped. Unless there is evidence of growth of a prolactinoma or related symptoms, there is no indication to continue dopamine agonist therapy after menopause ${ }^{32}$ The dopamine agonist dose be decreased after 2 or 3 years of normal prolactin levels and that therapy be stopped if the prolactin levels remain unchanged after 1 year at the reduced dose. The dose can be reduced by half over the course of 3 months and prolactin levels are measured monthly. After complete discontinuation of treatment, regular monitoring of clinical symptoms and prolactin levels is recommended. To detect early recurrence, prolactin levels should be measured monthly for the first 3 months and every 6 months thereafter.

\section{Conclusion}

Hyperprolactenemia is a common endocrine disorder in women having main consequences as amenorrhea and infertility and hence should be ruled out in all the cases of amenorrhea and infertility as restoration of ovulation is seen in $90 \%$ of cases if cause of hyperprolactenemia is identified and treated. Therefore, whenever women come with problems of infertility serum prolactin level must be estimated for proper management.

\section{References}

1. Luciano AA,Clinical presentation of hyperprolactinaemia. J. Reprod. Med 1999; 44 (suppl12):1085-1090

2. Patel SS,Bomigboye V,Hyperprolactenemia. J Obstet Gyneco 2007. 27(5):465-9(ISSN:01443615)

3. Peak Mann Mah,Jonathan Webster, hyperprolactenaemia: Etiology,diagnosis and management,Semin Reprod Med 20(4):365374,2002.

4. Draca S. Prolactin as an immmunoreactive agent. Immunology and Cell biology 1995; 73:481-3

5. Chikanza IC. Prolactin and Neuro immunomodulation in vitro and in vivo observation Ann Ny Acad Sec1999; 876:11930

6. Asa SL, Ezzat S. The pathogenesis of pituitary tumours. Nat Rev Cancer 2002; 2: 836-49. [Medline]

7. Omar Serri,Constance L. Chik,Ehud UR and Shereen Ezzat. Diagnosis and management of hyperprolactenemia . CMAJ. Sept. 2003; 169(6)

8. Craig Syrup, Hyperprolactenaemia, infertility \& beyond , 10-16,1990

9. Billar BM,Luciano A,Crosignani PG,et al. Guidelines for the diagnosis and treatment of hyperprolactenemia. 1999. 44(suppl12)):10751084

10. Donald Shenenberger,Hyperprolactenemia. eMedicine,Updated Mar12,2010

11. Braunwald, Fauci, Kasper, Hauser, Longo, Jameson. Harrison's Principles of Internal Medicine. 15th Edition. McGraw-Hill. 2001

12. Hammond and Talbort. A practical guide for the physician 3rd Ed. 103-105,1993

13. Ciccarelli E,Camanni F. Diagnosis and drug therapy of prolactinoma. Drugs 1996 Jun; 51 (6):954-65 
14. Schlechte, J, Sherman, B, Halmi, N, et al. Prolactin-secreting pituitary tumors in amenorrheic women: A comparative study. Endocr Rev 1980; 1:295.

15. Speroff L. Managing Galactorrheain pill patients. Contracept Technical update 1981 jun; 2(5):80-81

16. Billar BM. Hyperprolactenemia. Int J Fertil Womens Med 1999;44:74-77

17. Billar BM,Baum HB, Rosenthal DI,Saxe VC,Charpie PM,Klibanski A. Progressive trabecular osteopenia hyperprolactinemic amenorrhea. J Clin Endocrinol Metab 1992; 75:692-697

18. Biller BM. Diagnostic evaluation of hyperprolactenemia. J Reprod Med 1999Dec; 49(12 suppl):1095-9

19. Katarzyna Jaskiewicz,Jolanta KunertRodekRodek \& Marek Pawlikowstu. Is Metoclopromide test useful in differentiation of the clinical form of hyperprolactenemia? Endocrine. Abstracts (2008)16 p127

20. Le Moli R,Endert E ,Fliers E,Prummel MF,Wiersinga WM. Evaluation of endocrine tests: The TRH test in patients with hyperprolactenemia. Neth J Med 2003 Feb; 61(2):44-8

21. Molitch ME. Disorders of prolactin secretion. Endocrinal Metab Clin North Am 2001;30:585610

22. Gold stein M,Calne D,Lieberman A,Thomar M (eds):Ergot Compounds and Brain Function: Neuroendocrine and Neuropsychiatic Aspects. New York, Raven Press 1980; 125-189.

23. Moltich ME. Disorders of prolactin secretion. Endocrinol Metab Clin North Am 2001;30:585610
24. Webster J. A comparative review of the tolerability profiles of dopamine agonists in the treatment of hyperprolactenemia and inhibition of lactation. DRUG Saf1996; 14:228-238

25. Molitch ME. Management of prolactinomas during pregnancy. J Reprod Med 1999; 44(supp112):1121-112

26. Verhelst J,Abs R,Maiter D, et al. Cabergoline in the treatment of hyperprolactenemia:A study in 455 patients. J Clin Endocrinol Metab1999;84:2518-2522

27. Webster J. Piscitelli G,Polli A,Ferrari CI,Ismail I,Scanlon MF. A comparison of cabergoline and bromocriptine in hyperprolactinemic amenorrhea. Cabergoline Comparative STUDY Group. N Engl J Med 1994;331:904-909

28. Tyrrel JB,Lamborn KR,Hannegan LT,Applebury CB, Wilson CB. Transsphenoidal microsurgical therapy outcomes and long term results. Neurosurgery 1999;44:254-261

29. Wilson CB. Surgical management of pituitary tumors. J Clin Endocrinol Metab 1997;82:23812385

30. LandoltAM,Lomax N. Gamma knife radiosurgery for prolactinomas. J Neurosurg 2000; 93(suppl 3):14 -18

31. Webster J, Piscitelli G, Polli A, Ferrari CI, Ismail I, Scanlon MF. For the Cabergoline Comparative Study Group. A comparison of cabergoline and bromocriptine in the treatment of hyperprolactinemic amenorrhea. $N$ Engl J Med 1994; 331:904-9.

32. Passos VQ, Souza JJ, Musolino NR, Bronstein MD. Long-term follow-up of prolactinomas: normoprolactinemia after bromocriptine withdrawal. J Clin Endocrinol Metab 2002;87:3578-82. 